\title{
A Rare Association of Hunter Syndrome (Mucopolysaccharidosis Type II) with Ventricular Septal Defect
}

\section{Rahul Sinha $^{1 *}$, Rajveer Singh Beniwal ${ }^{2}$ and Sween Sheoran ${ }^{2}$}

${ }^{1}$ Pediatrics, Military Hospital, Jodhpur, India

${ }^{2}$ Radiology, Military, Hospital, Jodhpur, India

\begin{abstract}
Mucopolysaccharidosis (MPS) is a group of autosomal recessive metabolic disorders caused by the absence or malfunctioning of the lysosomal enzymes needed to break down molecules called glycosaminoglycans (GAGs). All the MPSs are inherited in an autosomal recessive fashion except Hunter syndrome (MPS type II), which is X-linked. Common clinical presentation includes facial dysmorphism, hepatosplenomegaly, joint stiffness and contractures, pulmonary dysfuction, myocardial enlargement and valvular dysfunction and neurological involvement. We report 17 yr old boy with features suggestive of Hunter syndrome associated with ventricular septal defect.
\end{abstract}

Keywords: Mucopolysaccaharidosis; Hunter; VSD; Glycosaminoglycans

\section{Introduction}

Mucopolysaccharidosis (MPS) is a group of autosomal recessive metabolic disorders caused by the absence or malfunctioning of the lysosomal enzymes needed to break down molecules called glycosaminoglycans(GAGs). Thesearelongchainsofsugarcarbohydrates in each cell that help build bone, cartilage, tendons, corneas, skin and connective tissues. Hunter syndrome, or mucopolysaccharidosis II (MPS II), is a serious genetic disorder that primarily affects males (X-linked recessive) [1]. It interferes with the body's ability to break down and recycle specific mucopolysaccharides, also known as glycosaminoglycans or GAG. Hunter syndrome is one of several related lysosomal storage diseases. Glycosaminoglycans (formerly called mucopolysaccharides) are also found in the fluid that lubricates joints. Eleven distinct single lysosomal enzyme deficiencies are known to cause 7 recognized phenotypes of MPS. All the MPSs are inherited in an autosomal recessive fashion except Hunter syndrome (MPS type II), which is X-linked. People with MPS either do not produce enough of one of the 11 enzymes required to break down these sugar chains into proteins and simpler molecules, or they produce enzymes that do not work properly. Over time, these GAGs collect in the cells, blood and connective tissues. This results in permanent, progressive cellular damage which affects the appearance, physical abilities, organ and system functioning and, in most cases, mental development. Common clinical presentation includes facial dysmorphism, hepatosplenomegaly, joint stiffness and contractures, pulmonary dysfuction, myocardial enlargement and valvular dysfunction and neurological involvement. We report $17 \mathrm{yr}$ old boy with features suggestive of Hunter syndrome associated with ventricular septal defect.

\section{Case Report}

17 years old boy presented to our OPD with history of shortness of breath on and off for last 6 yrs, unsteadiness of gait for last 4 yrs and excessive salivation for last 2 yrs. The antenatal and postnatal history was uneventful. There was also a history of joint pain on and off during the past two to three years. There was no history of constipation, diarrhea, vomiting, bleeding, jaundice, seizure, weight loss or loss of appetite or of consciousness. His bladder habit was normal. The scholastic performance was poor. There is no history of consanguineous marriage. There was young deaths in the family two girl children at 9 years and 14 years of age, male child at 8 yrs of age. The clinical features was similar to the index child however work up was not done for these children.
On examination his weight was $40 \mathrm{~kg}$ and head circumference of 55 $\mathrm{cm}$. The height was $140 \mathrm{~cm}$ with upper segment to lower segment ratio is 0.82 . He had a depressed nasal bridge, a short neck, coarse facial features, large tongue, long philtrum (Figure 1). His fundus appeared normal. The vital parameters was normal. His abdomen was soft with liver span $15 \mathrm{~cm}$. The heart sound was normal pansystolic murmur best heard at left sternal edge with grade $3 / 6$. Based on the clinical features possibility of MPS was kept and skeletal survey was done. Anteroposterior and lateral X-rays of the dorsolumbar spine showed anterior beaking at D12 and L1,2,3 with slender long pedicle and scoliosis at dorso lumbar spine with convexity to left (Figure 2a-2c). Anteroposterior and lateral X-rays of the skull showed an calvarial thickening and depressed bridge of nose

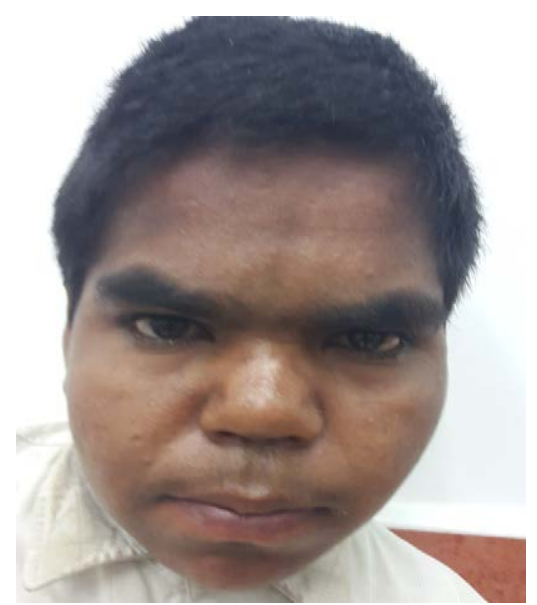

Figure 1: shows Coarse facial features with depressed nasal bridge, a short neck, long philtrum.

*Corresponding author: Rahul Sinha, Pediatrics, Military Hospital, Jodhpur, India; Tel: +918800630109, E-mail: drrahul_2000@yahoo.com

Received April 27, 2015; Accepted April 28, 2015; Published May 02, 2015

Citation: Sinha R, Beniwal RS, Sheoran S (2015) A Rare Association of Hunter Syndrome (Mucopolysaccharidosis Type II) with Ventricular Septal Defect. Human Genet Embryol 5: 127. doi:10.4172/2161-0436.1000127

Copyright: ( 2015 Sinha R, et al. This is an open-access article distributed under the terms of the Creative Commons Attribution License, which permits unrestricted use, distribution, and reproduction in any medium, provided the original author and source are credited. 
Citation: Sinha R, Beniwal RS, Sheoran S (2015) A Rare Association of Hunter Syndrome (Mucopolysaccharidosis Type II) with Ventricular Septal Defect. Human Genet Embryol 5: 127. doi:10.4172/2161-0436.1000127

Page 2 of 3

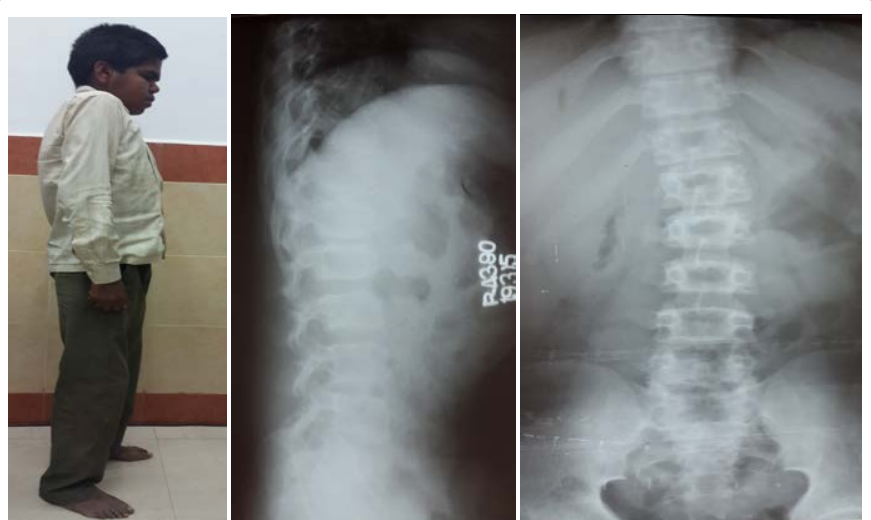

Figure 2: showing dorso lumbar scoliosis with anterior breaking at D12, L1,2,3 with ovoid shaped vertebra.

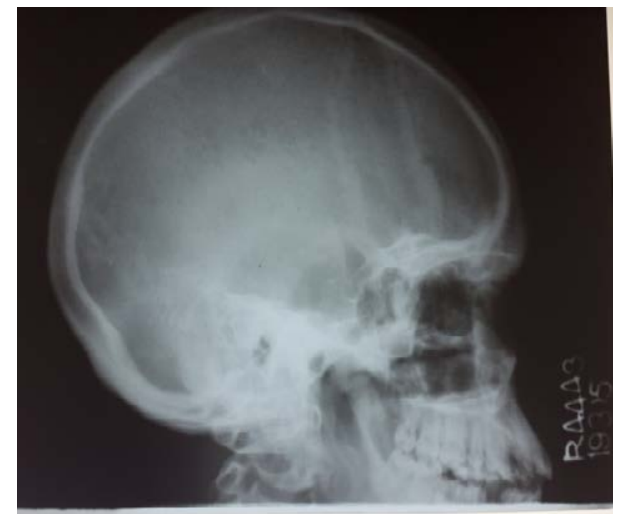

Figure 3: shows $X$ ray skull with calvarial thickening.

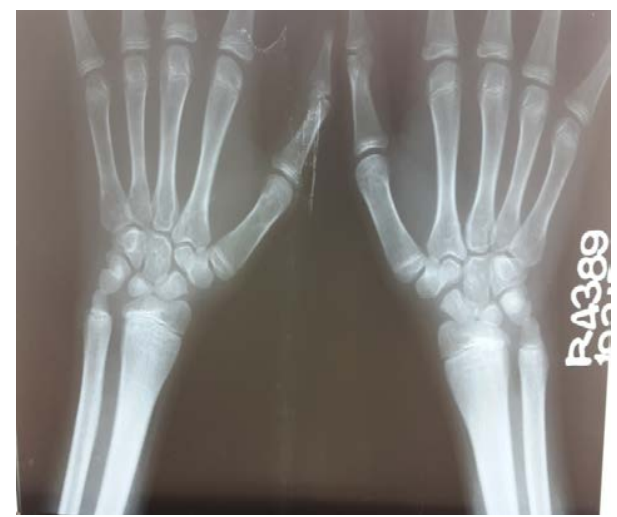

Figure 4: X ray wrist showing small carpal bone with pointed distal end of ulna

with frontal bossing with hypopneumatised mastoid (Figure 3). X-rays of both hands showed small carpal bone with pointed distal end of ulna. (Figure 4). The remaining bones and joints under view appeared normal. An anteroposterior X-ray showed his ribs as wide with tapered posterior ends (a paddle and/or spatulated appearance) (Figure 5). No parenchymal lesions were seen in visualized lung fluids. The $\mathrm{x}$ ray pelvis showed spina bifida at s1 with sclerosis and deformed femoral head and linear subcortical lucency in right femoral head (Figure 6). There was coxa valgus deformity bilaterally. The laboratory investigation showed Haemoglobin $14.1 \mathrm{gm} \%$, TLC 4400/cmm, DLC P51L39M5E5 with
Platelet 1.50 lacs $/ \mathrm{cmm}$. The renal function and liver function test was normal. The thyroid profile was normal. The lipid profile was normal. The ultrasonography of abdomen and kidney revealed hepatomegaly. The audiological test was normal. The Echocardiography showed left to right shunt at ventricular level with $9 \mathrm{~mm}$ VSD. There was no features suggestive of Pulmonary arterial hypertension. The MPS screening on urine was positive and enzyme assay revealed very low levels of iduronate-2-sulfatase.

Based on the clinical, biochemical and skeletal survey diagnosis of Mucopolysaccharidosis was made. The diagnosis of Hunter syndrome was made based on male child, no corneal clouding, skeletal survey and moderate mental retardation. The enzyme levels could not be done due to lack of facility for the same. This case report highlights the rare association of Hunter syndrome with VSD which is not reported in the literature.

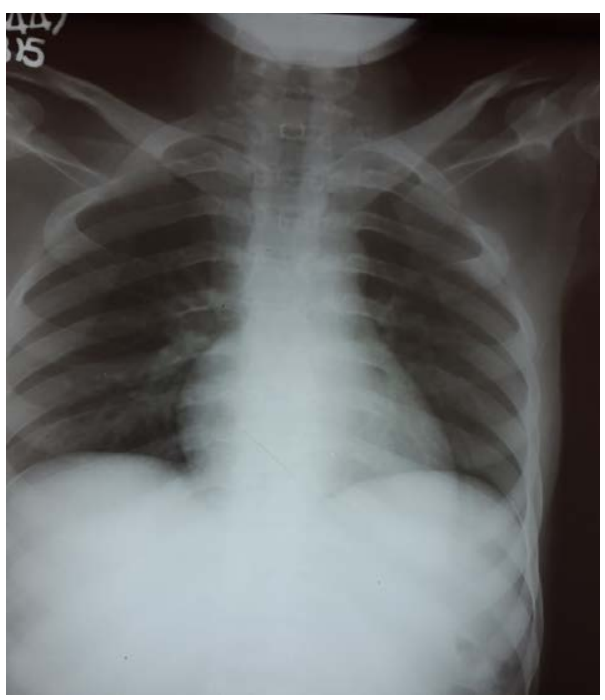

Figure 5: CXR showing ribs with tapered posterior ends (a paddle and/or spatulated appearance).

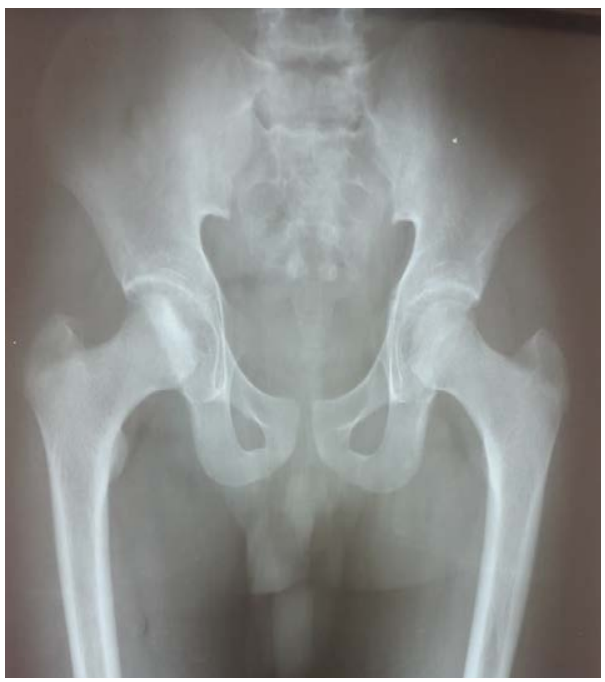

Figure 6: $\mathrm{X}$ ray pelvis showing spina bifida at $\mathrm{s} 1$ with sclerosis and deformed femoral head and linear subcortical lucency in right femoral head. 


\section{Discussion}

Mucopolysaccharidosis type II or Hunter syndrome is rare and is caused by a deficiency of iduronate-2-sulfatase. Hunter syndrome is one of the most common MPS with a prevalence of one in 170,000 male live births. MPS type II is classified into mild (type II, HB) and severe (type II, A) and this classification is based on the length of survival and the presence or absence of central nervous system (CNS) disease [2]. Patients typically appear normal at birth in both types. In the severe form the clinical features appear between two and four years of age while in the mild form the clinical features appear in the second decade of life. In the severe form there is severe mental retardation and loss of skills. Death usually occurs in the first or second decade of life and the main cause of death is obstructive airway disease or cardiac failure. In the milder form there is mild mental retardation but intelligence is normal, stature is near normal, and clinical features are less obvious and progress very slowly. Diagnosis is usually made in the second decade of life. Death usually occurs in the fourth decade and the main cause of death is cardiac failure.

The visible signs and symptoms of Hunter syndrome (MPS II) in younger people are usually the first clues leading to a diagnosis. The cardiac involvement includes deposition of storage material (glycosaminoglycan) occurs in the hearts of individuals with all forms of MPS Syndromes. The cardiac valves (usually mitral and aortic), the heart muscle itself (ventricular muscle) and the coronary arteries are characteristically affected in MPS Syndromes [3]. The onset and extent of cardiac involvement varies depending upon the type of MPS present and there is great variability even within specific MPS syndromes. Congenital abnormalities of the heart may also occasionally be present in children who have MPS Syndromes. Communications between the upper chambers (ASD) or great vessels (PDA) have been found in some children with MPS Syndromes. Sometimes extra electrical connections within the heart can cause an unusually fast heart beat (tachycardias). Usually these problems are treatable by conventional means. In general, the time of diagnosis usually occurs from about 2 to 4 years of age. Doctors may use laboratory tests to provide additional evidence that an MPS disorder is present, before making a definitive diagnosis by measuring the iduronate-2-sulfatase (I2S) enzyme activity. The most commonly used laboratory screening test for an MPS disorder is a urine test for GAG. It is important to note that the urine test for GAG can occasionally be normal and yet the child still may have an MPS disorder. A definitive diagnosis of Hunter syndrome is made by measuring I2S activity in serum, white blood cells, or fibroblasts from skin biopsy. In some people with Hunter syndrome, analysis of the I2S gene can determine clinical severity. Prenatal diagnosis is routinely available by measuring I $2 S$ enzymatic activity in amniotic fluid or in chorionic villus tissue.

Enzyme replacement therapy using idursulfase (Elaprase), a recombinant human $12 \mathrm{~S}$ produced in the human cell line, has been recently approved in the United States and the European Union for the management of MPS type II. Weekly intravenous infusion is given over three hours at a dose of $0.5 \mathrm{mg} / \mathrm{kg}$ diluted in saline. Bone marrow transplantation (BMT) and umbilical cord blood transplantation (UCBT) are definitive treatments for MPS. Apart from these, supportive management is very important. Physical therapy and daily exercise may improve mobility of joints. Blood transfusion, infection and nutritional management are also important in the management of MPS type II. It has been found that fibroblast from patients shows metachromatic cytoplasmic inclusions [4]. A mild form (MPS type IIB) is compatible with survival into adulthood, and reproduction is known to have occurred [5]. Six cases of this mild form of Hunter syndrome have been described, and the patients survived to the ages of 65 and 87 in two cases. Three of these affected men had children [6]. The enzyme deficient in this disorder is iduronate sulfatase, as described by Neufeld [7]. Occurrence of Mongolian spots in seven Japanese infants with Hunter syndrome before and after hematopoietic stem cell transplantation (HSCT) has been observed [8].

This case report highlights the rare association of Hunter syndrome with VSD which is not reported in the literature and it increases the phenotypic spectrum of the disease.

\section{Refrences}

1. Young ID, Harper PS (1982) "Incidence of Hunter's syndrome”. Hum Genet 60: 391-392.

2. Martin R, Beck M, Eng C, Giugliani R, Harmatz P, et al. (2008) Recognition and diagnosis of mucopolysaccharidosis II (Hunter syndrome). Pediatrics 121 377-386.

3. Dangel JH (1998) Cardiovascular changes in children with mucopolysaccharide storage diseases and related disorders - clinical and echocardiographic findings in 64 patients. Eur J Pediatr 157: 534-538.

4. Beck M, Steglich C, Zabel B, Dahl N, Schwinger E, et al. (1992) Deletion of the Hunter gene and both DXS466 and DXS304 in a patient with mucopolysaccharidosis type II. Am J Med Genet 44: 100-103.

5. Ben Simon-Schiff E, Bach G, Zlotogora J, Abeliovich D (1993) Combined enzymatic and linkage analysis for heterozygote detection in Hunter syndrome: identification of an apparent case of germinal mosaicism. Am J Med Genet 47: 837-842.

6. Berg K, Danes BS, Bearn AG (1968) The linkage relation of the loci for the Xm serum system and the X-linked form of Hurler's syndrome (Hunter syndrome). Am J Hum Genet 20: 398-401.

7. Daniele A, Natale P (1987) Hunter Syndrome: Presence of material crossreacting with antibodies against iduronate sulfatase. Human Genetics 75: 234-238.

8. Birot AM, Delobel B, Gronnier P, Bonnet V, Maire I, et al. (1996) A 5-megabase familial deletion removes the IDS and FMR-1 genes in a male Hunter patient. Hum Mutat 7: 266-268. 